\title{
Local uncertainty relations serving as measures of entanglement in a bipartite two-level system
}

\author{
Simon Samuelsson and Gunnar Björk \\ School of Information and Communication Technology, \\ Royal Institute of Technology (KTH), Electrum 229, SE-164 40 Kista, Sweden
}

(Dated: November 18, 2018)

\begin{abstract}
We comment on the recent suggestion to use a family of local uncertainty relations as a standard way of quantifying entanglement in two-qubit systems. Some statements made on the applicability of the proposed "measures" are overly optimistic. We exemplify how these specific "measures" fall short, and present a minor modification of the general theory which uses the same experimentally gathered information, but in a slightly different, better way.

PACS numbers: 03.67.Mn, 42.50.Dv, 03.65.Ta
\end{abstract}

\section{INTRODUCTION}

During the last few years an interesting approach of using sums of variances of local observables to probe for the existence of entanglement in N-level systems has been suggested. The original proposal by Hofmann and Takeuchi [1] has appealing features. Especially, the experimental effort needed to verify that a given source produces entangled states could be reduced substantially compared to the full state tomography [2, 3, 4, [5, 6, 7, 8], joint measurements of all members of an ensemble 9, 10, 11, or adaptive measurements [12, 13]. An extension of the theory has been made by Gühne [14]. In addition, Khan and Howell [15] have recently investigated the usefulness of the method for bipartite two-level systems, e.g., consisting of pairs of photons from a down-conversion source, entangled in polarization. Using inequalities based on sum-variances measured in different bases on the singlet state and on mixtures of the singlet state and two kinds of "noise", they state: "We suggest that these sum-variance inequalities could be very useful as standard entanglement measures for spin- $1 / 2$ systems."

In this brief exposé, we revisit the local uncertainty relations, or LURs, showing how bipartite entanglement 16 . can be revealed through local-observable correlations. Next, the proposed "measures" of entanglement are reiterated and commented, and it is shown that they are not suitable to quantify entanglement outside the frame set by Khan's and Howell's specific investigation. In this respect they are akin to entanglement witnesses [17, 18, 19, 20, 21, 22] in that a LUR can detect certain classes of entangled states, but most entangled states go undetected. (That is, violation of a LUR is a sure sign of entanglement, but nonviolation of a LUR implies nothing.) The general theory of LURs is then modified through the introduction of modified local uncertainty relations (MLURs) with improved characteristics.

\section{LOCAL UNCERTAINTY RELATIONS}

In the initial formulation by Hofmann and Takeuchi [1], LURs involving two systems, A and B, are considered. In order to construct a LUR, two sets of observables must be chosen, $\left\{\hat{A}_{i}\right\}$ and $\left\{\hat{B}_{i}\right\}$, acting solely on system A and B, respectively. Varying the state of the composite system, the sums of the local variances $\sum_{i} \delta^{2} \hat{A}_{i}$ and $\sum_{i} \delta^{2} \hat{B}_{i}$, where, e.g., $\delta^{2} \hat{A}_{i} \equiv\left\langle\hat{A}_{i}^{2}\right\rangle-\left\langle\hat{A}_{i}\right\rangle^{2}$, will each have a greatest lower bound, $U_{A}$ and $U_{B}$ respectively. The local uncertainty relation

$$
\sum_{i} \delta^{2}\left(\hat{A}_{i}+\hat{B}_{i}\right) \geq U_{A}+U_{B}
$$

is a relation that is supposed to hold for all mixtures of product states $\hat{\rho}=\sum_{k} p_{k} \hat{\rho}_{k}$, where $\sum_{k} p_{k}=1$ and $\hat{\rho}_{k}$ denote product states. The relation is obvious for product states, since in the expansion of the variance of a sum

$$
\delta^{2}\left(\hat{A}_{i}+\hat{B}_{i}\right)=\delta^{2} \hat{A}_{i}+\delta^{2} \hat{B}_{i}+2 C\left(\hat{A}_{i}, \hat{B}_{i}\right),
$$

the covariance term, $C\left(\hat{A}_{i}, \hat{B}_{i}\right)=\left\langle\hat{A}_{i} \hat{B}_{i}\right\rangle-\left\langle\hat{A}_{i}\right\rangle\left\langle\hat{B}_{i}\right\rangle$, is simply zero. The extension of (1) to hold for a statistical mixture of product states is easily justified through the general fact that if $\hat{\rho}=\sum_{k} p_{k} \hat{\rho}_{k}$, where $\hat{\rho}_{k}$ represents a product state $\forall k$, then

$$
\delta_{\hat{\rho}}^{2} \hat{S} \geq \sum_{k} p_{k} \delta_{\hat{\rho}_{k}}^{2} \hat{S}
$$


for an arbitrary observable $\hat{S}$, adding a subscript to the variances to make their state dependence explicit. The inequality (3) holds because $\delta_{\hat{\rho}}^{2} \hat{S}=\sum_{k} p_{k}\left\langle\left(\hat{S}-\langle\hat{S}\rangle_{\hat{\rho}}\right)^{2}\right\rangle_{\hat{\rho}_{k}}=\sum_{k} p_{k}\left\{\delta_{\hat{\rho}_{k}}^{2} \hat{S}+\left(\langle\hat{S}\rangle_{\hat{\rho}_{k}}-\langle\hat{S}\rangle_{\hat{\rho}}\right)^{2}\right\} \geq \sum_{k} p_{k} \delta_{\hat{\rho}_{k}}^{2} \hat{S}$.

When the systems are mixed or entangled, the covariance term in (2) is in general nonzero. If the LUR is to reveal nonseparability, a necessary condition is that at least one of the covariances between the systems are such that

$$
C\left(\hat{A}_{i}, \hat{B}_{i}\right)<0
$$

The covariance is bounded from below and above by the following relation:

$$
-\left(\delta^{2} \hat{A}_{i}+\delta^{2} \hat{B}_{i}\right) \leq 2 C\left(\hat{A}_{i}, \hat{B}_{i}\right) \leq \delta^{2} \hat{A}_{i}+\delta^{2} \hat{B}_{i}
$$

Interestingly, for any particular choice of a pair of observables $\hat{A}_{i}$ and $\hat{B}_{i}$, both bounds can be reached both with mixed separable, and pure entangled states. Hence, the essential difference between separable and entangled states cannot be distinguished by studying one observable pair alone, but lies rather in the existence of significant covariance between several pairs of observables simultaneously. The system of two spin-1/2 particles serves as an illustrative example. Measuring spin in the three cartesian directions $x, y$ and $z$, the revelation of maximum covariance in one direction implies, for separable states, no covariance in the other two directions. On the other hand, entangled states may possess maximum covariance [it saturates one of the bounds in (5)] in all three bases. The LURs exploit this characteristic feature of entanglement. However the important observation that follows from condition (4) is that a given LUR can only detect the entanglement for states with "appropriate" signs of the covariances.

Focussing on two-level systems, through polarization states of photon pairs, Khan and Howell investigated two closely related LURs based on polarization measurements in different bases:

$$
L_{2}=\delta^{2}\left(\hat{\sigma}_{A}+\hat{\sigma}_{B}\right)_{0 / 90}+\delta^{2}\left(\hat{\sigma}_{A}+\hat{\sigma}_{B}\right)_{45 / 135} \geq 2
$$

and

$$
L_{3}=\delta^{2}\left(\hat{\sigma}_{A}+\hat{\sigma}_{B}\right)_{0 / 90}+\delta^{2}\left(\hat{\sigma}_{A}+\hat{\sigma}_{B}\right)_{45 / 135}+\delta^{2}\left(\hat{\sigma}_{A}+\hat{\sigma}_{B}\right)_{R / L} \geq 4
$$

The lower bounds $L_{2} \geq 2$ and $L_{3} \geq 4$ are valid for any mixture of product states but may be violated for entangled states. The subscript $0 / 90$ refers to a measurement of horizontal-vertical polarization, while for $45 / 135$, the basis is rotated by 45 degrees. The third term to the left of the inequality sign of (7) denotes right- and left-handed circular polarization. In these two cases, $\hat{A}_{i}$ and $\hat{B}_{i}$ correspond to identical measurements on the two systems, so in the light of condition (4), negative covariances are favored. The optimum domain of entanglement verification for these LURs therefore necessarily involves the singlet state, exemplified, e.g., by a superposition of vertically (V) and horizontally $(\mathrm{H})$ linearly polarized photons: $\left|\psi^{-}\right\rangle=(|H, V\rangle-|V, H\rangle) / \sqrt{2}$. This state has the unique property among the maximally entangled states that a measurement of $\hat{A}_{i}+\hat{B}_{i}$, where $\hat{A}_{i}$ and $\hat{B}_{i}$ represent arbitrary, but identical polarization or spin measurements on the two particles, will always give the trivial outcome zero with certainty.

The only states previously studied [1, 14, 15] have density operators of the form

$$
\hat{\rho}=p\left|\psi^{-}\right\rangle\left\langle\psi^{-}\right|+(1-p) \hat{\chi}
$$

where $0 \leq p \leq 1$. These are clearly based on the singlet state, and the density operator $\hat{\chi}$ refers to either Werner noise or maximally polarized noise [16]. For these states both $L_{2}$ and $L_{3}$, the latter in particular, shows high sensitivity in detecting entanglement, compared to the corresponding Bell measurement. Analytically it has been shown that entanglement exists for values of $p$ within the range $1 / 3<p \leq 1$ for Werner noise and $0<p \leq 1$ for maximally polarized noise. $L_{3}$ drops below its lower bound 4 precisely for these values of $p$.

The application of these particular LURs has been extended further by letting the magnitude of the violation constitute a measure of the degree of entanglement for a given state. Khan and Howell [15] even suggest application beyond the states of the form (8) concerning $L_{3}$ by claiming: "This measurement therefore should be ideal for standardizing entanglement measures in spin-1/2 systems". This extended use, however, requires that the behavior of the LURs obey certain criteria. One important such is invariance under local unitary transformations. However, such transformations are capable of changing both the amount and the sign of the covariance (due to entanglement) in different bases, while they are unable to alter the degree of entanglement in the system. As shown by Eq. (2), a single LUR is highly sensitive to the kind of covariances present. This unfortunately makes them unsuitable as entanglement measures for general states.

As an example, the four Bell states

$$
\left|\psi^{ \pm}\right\rangle=\frac{1}{\sqrt{2}}(|H, V\rangle \pm(|V, H\rangle) \quad \text { and }
$$




$$
\left|\phi^{ \pm}\right\rangle=\frac{1}{\sqrt{2}}(|H, H\rangle \pm(|V, V\rangle)
$$

can be considered. Since each Bell state can be transformed by a local unitary transformation into any of the other, a functional associating each state in the Hilbert space with a real value can only be considered a reasonable measure of entanglement if all the Bell states are assigned the same values. This is not the case for the "measures" $L_{2}$ and $L_{3}$. Only the singlet state gives the desired values $L_{2}=L_{3}=0$ of maximum violation, and for the other three Bell states, $L_{2}=4$ and $L_{3}=8$, which is not even close to violating the corresponding LURs, in spite of the fact that these states are maximally entangled. The reason for this is that these Bell states have negative polarization covariance in one basis only, and positive covariance in the other two. Consequently, $L_{2}$ and $L_{3}$ will only detect entangled states belonging to the class defined by Eq. (8).

\section{MODIFIED LOCAL UNCERTAINTY RELATIONS}

Fortunately, with a slight modification the LURs can be restated in a manner that makes them sensitive only to the magnitude, and not to the sign, of the covariances, requiring no further experimental effort. For ordinary LURs, the expression $\sum_{i} \delta^{2} \hat{A}_{i}+\delta^{2} \hat{B}_{i}+2 C\left(\hat{A}_{i}, \hat{B}_{i}\right)$ is bounded below, and derives from expanding (1) using (2). Now forcing all the covariance terms to contribute negatively by subtracting the correlation moduli instead, we arrive at the modified LUR, or MLUR,

$$
\sum_{i} \delta^{2} \hat{A}_{i}+\delta^{2} \hat{B}_{i}-2\left|C\left(\hat{A}_{i}, \hat{B}_{i}\right)\right|=\sum_{i} \delta^{2}\left(\hat{A}_{i}+\hat{B}_{i}\right)-4 \operatorname{Max}\left\{0, C\left(\hat{A}_{i}, \hat{B}_{i}\right)\right\} \geq U_{A}+U_{B}
$$

In any measurement where variances of the form $\delta^{2}\left(\hat{A}_{i}+\hat{B}_{i}\right)$ are determined, the rates of incidence necessary to calculate $\delta^{2} \hat{A}_{i}$ and $\delta^{2} \hat{B}_{i}$ are also directly available. Therefore, the covariances in (9) are at hand through Eq. (2). A vital question is whether the lower bound for separable states, $U_{A}+U_{B}$, is still valid for the MLURs, as already implied. To prove that this is indeed the case we consider the first sum in (9) for a mixture of product states $\hat{\rho}$. Since the covariance is linear in both arguments we can choose a sign $\pm_{\hat{\rho}}$ preceding $\hat{B}_{i}$, depending on the state $\hat{\rho}$, so that each term in (9) can be written as

$$
\delta_{\hat{\rho}}^{2} \hat{A}_{i}+\delta_{\hat{\rho}}^{2}\left( \pm_{\hat{\rho}} \hat{B}_{i}\right)+2 C_{\hat{\rho}}\left(\hat{A}_{i}, \pm_{\hat{\rho}} \hat{B}_{i}\right)=\delta_{\hat{\rho}}^{2}\left(\hat{A}_{i} \pm_{\hat{\rho}} \hat{B}_{i}\right) \geq \sum_{k} p_{k} \delta_{\hat{\rho}_{k}}^{2}\left(\hat{A}_{i} \pm_{\hat{\rho}} \hat{B}_{i}\right)=\sum_{k} p_{k} \delta_{\hat{\rho}_{k}}^{2}\left(\hat{A}_{i}+\hat{B}_{i}\right) .
$$

The inequality is nothing but (3), and the last equality holds only because $\hat{\rho}_{k}$ denotes a product state, and the covariance term is zero for product states. Thus, the inequality (9) becomes

$$
\sum_{i} \delta_{\hat{\rho}}^{2} \hat{A}_{i}+\delta_{\hat{\rho}}^{2} \hat{B}_{i}-2\left|C_{\hat{\rho}}\left(\hat{A}_{i}, \hat{B}_{i}\right)\right| \geq \sum_{i} \sum_{k} p_{k} \delta_{\hat{\rho}_{k}}^{2}\left(\hat{A}_{i}+\hat{B}_{i}\right) \geq \sum_{k} p_{k}\left(U_{A}+U_{B}\right)=U_{A}+U_{B}
$$

We conclude that the modification of a LUR into an MLUR does not alter the greatest lower bound for separable states.

This modification of the theory inherits all the appealing characteristics of the LURs, while extending the subset of entangled states that can be detected with any given LUR, as seen in Fig. 1. The ideal case is maximum violation of the lower bound 4, fulfilled by both expressions for the singlet state $\left|\psi^{-}\right\rangle$. However, for the local unitary transformation $\hat{U}_{1}\left|\psi^{-}\right\rangle=\left(i\left|\psi^{+}\right\rangle+\left|\psi^{-}\right\rangle+\left|\phi^{+}\right\rangle-i\left|\phi^{-}\right\rangle\right) / 2, M L_{3}$ experiences its worst-case scenario under all such transformations, where all three covariances vanish and $M L_{3}=L_{3}=6$. On the other hand, the improved characteristics of MLURs over LURs are illustrated by the states $\hat{U}_{2}\left|\psi^{-}\right\rangle=\left(-i \sqrt{3}\left|\psi^{+}\right\rangle+\left|\psi^{-}\right\rangle\right) / 2$ and $\hat{U}_{3}\left|\psi^{-}\right\rangle=\left|\psi^{+}\right\rangle$for which $L_{3}$ is unable to detect entanglement. $M L_{3}$ verifies the existing maximum entanglement through partial and full violation, respectively, of its bound. The explicit form of the local unitary transformations used above are

$$
\begin{gathered}
\hat{U}_{1}=\frac{1}{2}\left(\begin{array}{cc}
1+i & -1+i \\
1+i & 1-i
\end{array}\right) \otimes\left(\begin{array}{ll}
1 & 0 \\
0 & 1
\end{array}\right), \\
\hat{U}_{2}=\frac{1}{2}\left(\begin{array}{cc}
1-i \sqrt{3} & 0 \\
0 & 1+i \sqrt{3}
\end{array}\right) \otimes\left(\begin{array}{ll}
1 & 0 \\
0 & 1
\end{array}\right), \quad \text { and }
\end{gathered}
$$




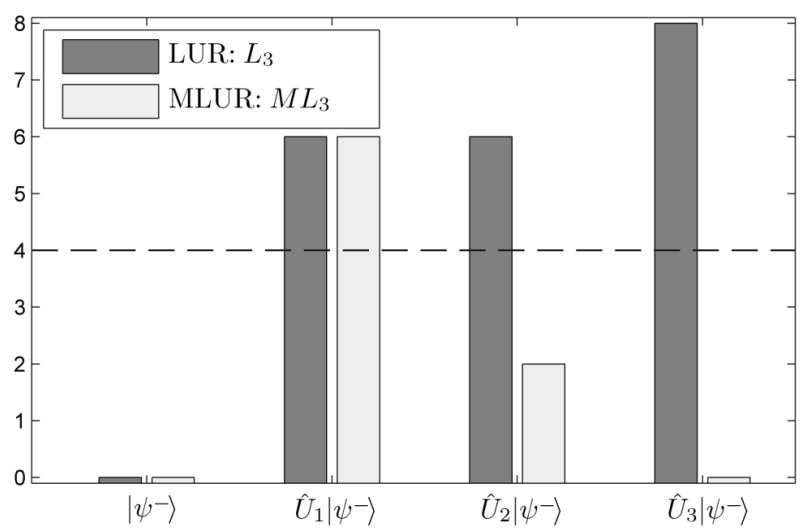

FIG. 1: The difference in behavior of a LUR and an MLUR, exemplified through $L_{3}$ and $M L_{3}$, for fully entangled states.

$$
\hat{U}_{3}=\left(\begin{array}{cc}
1 & 0 \\
0 & -1
\end{array}\right) \otimes\left(\begin{array}{ll}
1 & 0 \\
0 & 1
\end{array}\right)
$$

The modification of $L_{3}$ into $M L_{3}$ amounts to treating all Bell states in the same manner, thus avoiding the troublesome behavior mentioned earlier. This new MLUR is in fact maximally violated for all four Bell states. As a consequence, this generalizes the results regarding entanglement verification for states of the form (8). The MLUR is similarly violated for the kinds of noise studied, if the singlet states are replaced by any other Bell state, that is $\hat{\rho}=p|\operatorname{Bell}\rangle\langle\operatorname{Bell}|+(1-p) \hat{\chi}$.

The concept of MLURs also provides useful insight in the way $L_{2}$ detects entanglement. Given any state, $L_{2}$ is always non-negative and bounded above by the inequalities $0 \leq L_{2} \leq 8$, and the existence of entanglement is ensured for $0 \leq L_{2}<2$. However, significant covariances in the two particular bases used must also be present in order to find $L_{2}$ within the range $6<L_{2} \leq 8$. By constructing $M L_{2}$, the MLUR corresponding to $L_{2}$, all states satisfying this condition on $L_{2}$ are found to violate the MLUR. That is, $M L_{2}<2$ if $L_{2}>6$. In order to prove this statement, we first note that $\delta^{2} \hat{A}_{i} \leq 1$ for any polarization measurement performed on one of the photons. The explicit expressions for $M L_{2}$ and $L_{2}$ each contain four such variances. Thus, using the definitions of $L_{2}$ and $M L_{2}$ through the equality in (6) and the left-hand sum in (9), respectively,

$$
M L_{2} \leq 4-\left|2 C\left(\hat{\sigma}_{A}, \hat{\sigma}_{B}\right)_{0 / 90}+2 C\left(\hat{\sigma}_{A}, \hat{\sigma}_{B}\right)_{45 / 135}\right| \leq 4-\left(L_{2}-4\right)<2,
$$

keeping in mind that $L_{2}>6$. This fact consequently introduces an upper bound in the LUR in Eq. (6), so that $2 \leq L_{2} \leq 6$ for separable states. This would be of immediate use for anyone clinging to $L_{2}$. However, we advocate the use of $M L_{2}$ instead of $L_{2}$. The reason for this is simply that the MLUR can detect entanglement for an even larger class of states, namely those having significant covariances with opposite signs. For such states $L_{2}$ typically fails to detect entanglement. The state $\hat{U}_{2}\left|\psi^{-}\right\rangle=\left(-i \sqrt{3}\left|\psi^{+}\right\rangle+\left|\psi^{-}\right\rangle\right) / 2$ exemplifies this, having $2 C\left(\hat{\sigma}_{A}, \hat{\sigma}_{B}\right)_{0 / 90}=-2$ and $2 C\left(\hat{\sigma}_{A}, \hat{\sigma}_{B}\right)_{45 / 135}=1$, since $L_{2}=3$, providing no information, but $M L_{2}=1$, verifying present entanglement.

It must be stressed, however, that not even the suggested MLURs should be considered as measures of entanglement for general states. There are still certain local unitary transformations of the Bell states, e.g. $\hat{U}_{1}\left|\psi^{-}\right\rangle$in Fig. 1 , such that the transformed states do not reveal their entanglement through the particular observables used in e.g. $L_{3}$ and $M L_{3}$. Rather, LURs and MLURs should be used with the same caution as we do with entanglement witnesses. MLURs cannot detect all kinds of bipartite entanglement, but on the other hand, the necessary measurements can be performed with much less resources than, e.g., a full state tomography (that, on the contrary, will detect entanglement, irrespective of its type).

\section{CONCLUSIONS}

Conclusively, using the notion of covariance, we have shown how entangled states can violate LURs. However it also reveals the dependence on the sign of the covariance, causing an unnatural asymmetry in the way Bell states are treated by the proposed measures $L_{2}$ and $L_{3}$ [15]. Using this information, the general theory of LURs has been refined by introducing MLURs that process identically the same measurement data in a more efficient way. Their advantage lies in the fact that they can detect entanglement for a larger class of states than the corresponding LURs, without any additional measurements. In the specific case of $L_{3}$ and $M L_{3}$, their relative ease to measure is at the expense 
of both being sensitive to local unitary transformations. Computer simulations show better behavior of $M L_{3}$ than $L_{3}$, although it can be deceptive to regard any of them as a general measure of entanglement. Instead, $M L_{3}$ should be viewed as a measure of "Bell-type" entanglement, which is of substantial interest in characterizing the quality of entanglement from different sources.

\section{Acknowledgments}

This work was partially supported by the Swedish Foundation for Strategic Research (SSF) and the Swedish Research Council (VR).

[1] H. F. Hofmann and S. Takeuchi, Phys. Rev. A 68, 032103 (2003).

[2] K. Vogel and H. Risken, Phys. Rev. A 40, 2847 (1989).

[3] U. Leonhardt, Phys. Rev. Lett. 74, 4101 (1995); Phys. Rev. A 53, 2998 (1996).

[4] D. T. Smithey, M. Beck, M. G. Raymer, and A. Faridani, Phys. Rev. Lett. 70, 1244 (1993).

[5] D. Liebfried, D. M. Meekhof, B. E. King, C. Monroe, W. M. Itano, and D. J. Wineland, Phys. Rev. Lett. 774281 (1996).

[6] A. G. White, D. F. V. James, P. H. Eberhard, and P. G. Kwiat, Phys. Rev. Lett. 83, 3103 (1999).

[7] A. I. Lvovsky, H. Hansen, T. Aichele, O. Benson, J. Mlynek, and S. Schiller, Phys. Rev. Lett. 87, 050402 (2001).

[8] M. Barbieri, F. De Martini, G. Di Nepi, and P. Mataloni, quant-ph/0307003 G. Di Nepi, F. De Martini, M. Barbieri, and P. Mataloni, quant-ph/0307204

[9] A. Peres and W. K. Wootters, Phys. Rev. Lett. 66, 1119 (1991).

[10] S. Massar and S. Popescu, Phys. Rev. Lett. 74, 1259 (1995).

[11] R. Derka, V. Bužek, and A. Ekert, Phys. Rev. Lett. 80, 1571 (1998).

[12] D. Brody and B. Meister, Phys. Rev. Lett. 76, 1 (1996).

[13] D. G. Fisher, S. H. Kienle, and M. Freyberger, Phys. Rev. A 61, 032306 (2000).

[14] O. Gühne, Phys. Rev. Lett. 92, 117903 (2004).

[15] I. A. Khan and J. C. Howell, Phys. Rev. A 70, 062320 (2004).

[16] A. Peres, Phys. Rev. Lett. 77, 1413 (1996).

[17] M. Horodecki, P. Horodecki, and R. Horodecki, Phys. Lett. A 223, 1 (1996).

[18] M. Lewenstein et al., Phys. Rev. A 62, 052310 (2000).

[19] M. Lewenstein et al., Phys. Rev. A 63, 044304 (2001).

[20] B. Terhal, Phys. Lett. A 271, 319 (2000).

[21] D. Bruß et al., J. Mod. Opt. 49, 1399 (2002).

[22] M. Bourennane et al., Phys. Rev. Lett. 92, 087902 (2004). 